\title{
UNIFICATION OF GRAVITATIONAL AND STRONG NUCLEAR FIELDS
}

\author{
M. W. Evans \\ Alpha Institute for Advanced Study \\ E-mail: emyrone@aol.com
}

Received 19 October 2003

Using the recently derived Evans wave equation of unified field theory the strong nuclear field is described with an $\mathrm{SU}(3)$ representation of the gravitational field and the Gell-Mann color triplet is derived from general relativity as a three-spinor eigenfunction of the Evans wave equation.

Key words: Evans wave equation, strong field, gravitational field, GellMann color triplet, field unification.

\section{INTRODUCTION}

Recently a wave equation for unified field theory has been derived [1-3] from a lemma of Cartan-Riemann differential geometry [4, 5] and used to give the first self consistent unified description of the gauge invariant gravitational and electromagnetic fields. The former is the Riemann form and the latter is the torsion form. One form is the Hodge dual of the other. The theory has been shown [1-3] to reduce to all the main equations of physics in the appropriate limits, these equations include: the four Newton laws; the Schrödinger equation; the Dirac equation; the d'Alembert equation; the Poisson equations of dynamics and electrostatics; and the correct generally covariant form of the Maxwell-Heaviside field equations. The latter are referred to as " $\mathrm{O}(3)$ electrodynamics" because the symmetry group of the underlying gauge field theory is $\mathrm{O}(3)$. The theory of $\mathrm{O}(3)$ electrodynamics has been extensively tested against experimental data, and found experimentally to have numerous advantages over the Maxwell-Heaviside field theory [6-8]. It has been inferred that in order to unify gravitation and elec- 
tromagnetism within the theory of general relativity, electrodynamics must have a gauge symmetry higher than $U(1)$ [6-8]. This inference produces the fundamental Evans-Vigier magnetic field, which reaches mega-gauss in the inverse Faraday effect [9] of under dense plasma, and is now a routine observable. The theory reduces to two MaxwellHeaviside equations for transverse plane waves, but in addition gives the observable Evans-Vigier field, governed by a third field equation [6-8] not present in Maxwell-Heaviside theory. The unified field theory therefore has all the hallmarks of a major paradigm shift in physics. The inter-relation between the gravitational and electromagnetic field is given, for example, by the first and second Bianchi identities of differential geometry. These identities inter-relate the Riemann and torsion forms, which are defined in terms of the spin connection and the tetrad by the second and first Maurer-Cartan structure relations, respectively. It has been inferred that the Riemann form is the Hodge dual of the torsion form, and that spin connection is the Hodge dual of the tetrad. In the condensed notation of differential geometry the first and second Maurer-Cartan structure relations are, respectively:

$$
\begin{aligned}
& T^{a}=D \wedge q^{a}, \\
& R_{b}^{a}=D \wedge \omega_{b}^{a},
\end{aligned}
$$

where $R_{b}^{a}$ is the Riemann form and $T^{a}$ the torsion form. The symbol $D^{\wedge}$ denotes exterior covariant derivative, and $\omega_{b}^{a}$ and $q^{a}$ are, respectively, the spin connection and the tetrad. The first and second Bianchi identities are the homogeneous field equations of unified field/matter theory and are, respectively:

$$
\begin{aligned}
& D \wedge T^{a}:=0, \\
& D \wedge R_{b}^{a}:-0 .
\end{aligned}
$$

The first and second Evans duality equations state that there exist the Hodge duality relations

$$
\begin{aligned}
R_{b}^{a} & =\varepsilon_{b c}^{a} T^{c}, \\
\omega_{b}^{a} & =\varepsilon_{b c}^{a} q^{c},
\end{aligned}
$$

where $\varepsilon_{b c}^{a}$ is the appropriate Levi-Civita symbol in the well defined $[4,5]$ orthonormal space of the tetrad. by

In this notation, the Maxwell-Heaviside field theory is described

$$
\begin{aligned}
& F=d \wedge A, \\
& d \wedge F:=0,
\end{aligned}
$$

where $F$ is the gauge invariant electromagnetic field (a scalar-valued two-form), and where $A$ is the potential field (a scalar-valued oneform). In generally covariant unified field theory [1-3] $F$ becomes directly proportional to the torsion form $T^{a}$ (a vector valued two form) 
and $A$ becomes directly proportional to the tetrad $q^{a}$ (a vector-valued one-form). The exterior derivative $d^{\wedge}$ becomes the covariant exterior derivative $D^{\wedge}$. The inhomogeneous Maxwell-Heaviside field equation is

$$
d \wedge^{*} F=J
$$

where ${ }^{*} F$ is the Hodge dual of $F$ and where $J$ is a scalar-valued threeform, the current form. In unified field theory, Eq. (9) is developed into the first and second Evans inhomogeneous field equations

$$
\begin{aligned}
& D \wedge^{*} T^{a}=J^{a}, \\
& D \wedge^{*} R_{b}^{a}=J_{b}^{a},
\end{aligned}
$$

where $J^{a}$ is a vector valued three form, and $J_{b}^{a}$ is a tensor-valued three form, where ${ }^{*} T^{a}$ is the Hodge dual of $T^{a}$, defined by

$$
{ }^{*} T^{a \mu \nu}=\frac{1}{2} \varepsilon^{\mu \nu \rho \sigma} T_{\rho \sigma}^{a},
$$

and where ${ }^{*} R_{b}^{a}$ the Hodge dual of $R_{b}^{a}$, defined by

$$
{ }^{*} R_{b}^{a \mu \nu}=\frac{1}{2} \varepsilon^{\mu \nu \rho \sigma} R_{b \rho \sigma}^{a} .
$$

Equations (10) and (11) govern the interaction of the gauge invariant fields ${ }^{*} T^{a}$ and ${ }^{*} R_{b}^{a}$, respectively, with matter fields in unified field theory.

It can be seen that there is no longer a distinction between the gravitational and electromagnetic fields and they are unified with differential geometry. The potential field (Feynman's "universal influence" [10]) is developed into the tetrad, which is in turn governed by the Evans lemma of differential geometry:

$$
D D=d D:=\square-R,
$$

where $\square$ is the d'Alembertian operator in Euclidean spacetime, and where $\mathrm{R}$ is scalar curvature in differential geometry. The lemma (14) is the subsidiary proposition which leads to the Evans wave equation of differential geometry, the eigenequation

$$
\begin{gathered}
\square q^{a}=R q^{a}, \\
(\square+k T) q^{a}=0 .
\end{gathered}
$$

Combining Eq. (15) with the Evans field equation [1-3]

$$
R q^{a}=-k T q^{a}
$$


gives the Evans wave equation (16) of generally covariant unified field theory. Equation (16) completes Einstein's theory of general relativity of the gravitational field and develops it into a generally covariant unified field theory of all known radiation and matter fields.

The torsion form is the commutator of tetrads [1-3]:

$$
T^{c}=R q^{a} \wedge q^{b}
$$

and, using Eq. (5), the Riemann form is also defined in terms of a commutator of tetrads:

$$
R_{b}^{a}=\epsilon_{b c d}^{a} R q^{c} \wedge q^{d} .
$$

In this paper, we use Eq. (16) to derive the Gell-Mann quark color triplet from differential geometry in non-Euclidean spacetime, thus unifying the theory of the strong field and the theory of the gravitational field.

\section{DERIVATION OF THE QUARK COLOR TRIPLET}

It is well known that the quark Lagrangian is invariant under the $\mathrm{SU}(3)$ transformation [10], where $q$ is the quark color triplet

$$
\begin{gathered}
q \rightarrow M q, \\
q=\left(\begin{array}{c}
q_{R} \\
q_{W} \\
q_{B}
\end{array}\right)
\end{gathered}
$$

and a three spinor. In unifying the gravitational and strong fields it is shown in this section that $q$ is a manifestation of differential geometry, an eigenfunction of Eq. (16). Therefore the equation governing the strong force is

$$
(\square+k T) q=0,
$$

and $q$ is the gluon wavefunction, the eigenfunction of the quantized strong field. The corresponding wave equation for the gravitational field is

$$
(\square+k T) q_{\mu}^{a}=0,
$$

where $q_{\mu}^{a}$ is the tetrad. Therefore, if we can express Eq. (22) in terms of Eq. (23), we can unify the strong and gravitational fields.

In order to achieve this unification, note that $M$ in Eq. (20) is a unitary, orthogonal matrix of the $\mathrm{SU}(3)$ group representation of space, a $3 \times 3$ generalization of the $2 \times 2$ complex Pauli matrices [10] of the 
SU(2) group representation of space. Only unitary, orthogonal matrices form groups; Hermitian matrices do not form groups [10]. Therefore $M$ must be either an $\mathrm{O}(3)$ or $\mathrm{SU}(3)$ representation of space [10]. The two representations are representations of the same base manifold, so there must be relations between elements of $\mathrm{SU}(3)$ (components of the three spinor) and those of $\mathrm{O}(3)$ (components of the tetrad). In the unified field theory summarized in Sec. 1, this geometrical relation unifies the strong and gravitational fields, and shows that the strong field is simply the gravitational field in an $\mathrm{SU}(3)$ representation of space. Quarks have not yet been observed individually, so it remains to be seen whether the $\mathrm{SU}(3)$ representation has a physical meaning akin to the half integral spin of a fermion in the $\mathrm{SU}(2)$ representation. In other words the gravitational field can always be represented mathematically in $\mathrm{SU}(3)$ form, but it remains to be seen whether this has any meaning in physics, because individual quarks have never been observed. Indeed the standard model goes so far as to suggest that quarks cannot be observable, and "self-destructs" because quarks cannot be physical if they are unobservable. The major inference in Eq. (22) is that the quark color triplet is derived from a tetrad, and it should be noted that Eq. ( 22) is valid in non-Euclidean spacetime, and is a generally covariant description of the strong field.

In the standard model the $\mathrm{SU}(3)$ group is chosen to represent the strong field for experimental reasons [10], but these reasons do not include the observation of individual quarks. The SU(3) symmetry matrix $M$ is the unitary complex $3 \times 3$ matrix with unit determinant defined by

$$
M M^{\dagger}=1, \quad \operatorname{det} M=1 .
$$

It follows that $M$ is $3 \times 3$ matrix with eight independent parameters, which are the eight group generators denoted [10] by $\lambda_{n} / 2$. The corresponding generators in the $\mathrm{SU}(2)$ representation are the three Pauli matrices. Elementary particles in the standard model are thought to be composite states of quarks, and there are thought to be six quarks: $u, d, s, c, t$, and $b$. Each quark has spin half. Baryons and mesons are composite states of quarks, and in the standard model only these composite states are thought to be observable as elementary particles. Pions, for example, are thought to be three members of a supermultiplet of eight, and the strong interaction is thought to be an interaction between quarks mediated by a massless gluon field. All this is in the context of special relativity. There is no concept in the standard model of the strong field being derivable from the gravitational field, and so the strong field is not generally covariant, a major weakness of the standard model. In the unified field theory represented by Eqs. (22) and (23) the strong field is a manifestation of the gravitational field using $\mathrm{SU}(3)$ representation for the base manifold. The quark color triplet is simply a three spinor equivalent of the tetrad used to describe the gravitational field. The index of the orthonormal tangent space of the 
tetrad becomes the internal index of the $\mathrm{SU}(3)$ gauge group representation of the strong field. In the standard model the tangent space is a physical space but the internal space of gauge theory is an abstract space. In the unified field theory outlined in Sec. 1 the hitherto abstract internal space of gauge theory is given a physical meaning for the first time. Despite the fact that quarks are unobservable in the standard model, they are thought to possess a quantum number with three degrees of freedom, and these are the components of the three spinor (21): red, white and blue.

(Similarly the weak nuclear field in the standard model is thought to derive from a $\mathrm{SU}(2)$ symmetry gauge field theory. However the theory of the weak nuclear field is again a theory of special relativity, and spontaneous symmetry breaking is needed to provide the bosons of the weak field with mass. Unification of the weak and electromagnetic fields is standard-modeled in such a way that the electromagnetic field photons remain massless. This is essentially an empirical model with adjustable parameters. There is no experimental evidence for the Higgs boson for example, and so there is no evidence for spontaneous symmetry breaking, a cornerstone of the standard model. In the unified field theory of Sec. 1, the weak field is the electromagnetic field in $\mathrm{SU}(2)$ representation, and the mass of the three weak field bosons is represented by the limit of $T$ in special relativity, a mass density. Each boson of the weak field has its individual mass, determined experimentally.)

Therefore, in order to describe quarks in general relativity, nonEuclidean space-time must be expressed in SU(3) representation using the tetrad as a starting point. Similarly the tetrad is used [4,5] as the starting point for the recent derivation [11] of the Dirac equation from the more general Evans wave Eq. (16). The quarks (albeit unobservable by hypothesis in the standard model) of the strong nuclear field and the bosons of the weak nuclear field both become quanta of the unified field from the Evans wave equation. Ultimately the quarks are $\mathrm{SU}(3)$ representations of quantized gravitation from Eq. (16), and the weak nuclear field bosons are $\mathrm{SU}(2)$ representations of the electromagnetic field. Both the gravitational and the electromagnetic field spring from the tetrad, so we have built field unification with differential geometry.

The eight $\mathrm{SU}(3)$ matrices [10] are generalizations of the familiar Pauli matrices

$$
\sigma^{1}=\left(\begin{array}{ll}
0 & 1 \\
1 & 0
\end{array}\right), \quad \sigma^{2}=\left(\begin{array}{cc}
0 & -i \\
i & 0
\end{array}\right), \quad \sigma^{3}=\left(\begin{array}{cc}
1 & 0 \\
0 & -1
\end{array}\right)
$$

as follows:

$$
\sigma^{1} \rightarrow \lambda_{1}=\left(\begin{array}{lll}
0 & 1 & 0 \\
1 & 0 & 0 \\
0 & 0 & 0
\end{array}\right), \lambda_{4}=\left(\begin{array}{lll}
0 & 0 & 1 \\
0 & 0 & 0 \\
1 & 0 & 0
\end{array}\right), \lambda_{6}=\left(\begin{array}{lll}
0 & 0 & 0 \\
0 & 0 & 1 \\
0 & 1 & 0
\end{array}\right),
$$




$$
\begin{aligned}
\sigma^{2} \rightarrow \lambda_{2} & =\left(\begin{array}{ccc}
0 & -i & 0 \\
i & 0 & 0 \\
0 & 0 & 0
\end{array}\right), \lambda_{5}=\left(\begin{array}{ccc}
0 & 0 & -i \\
0 & 0 & 0 \\
i & 0 & 0
\end{array}\right), \lambda_{7}=\left(\begin{array}{ccc}
0 & 0 & 0 \\
0 & 0 & -i \\
0 & i & 0
\end{array}\right), \\
\sigma^{3} \rightarrow \lambda_{3} & =\left(\begin{array}{ccc}
1 & 0 & 0 \\
0 & -1 & 0 \\
0 & 0 & 0
\end{array}\right), \lambda_{8}=\frac{1}{\sqrt{3}}\left(\left(\begin{array}{ccc}
1 & 0 & 0 \\
0 & 0 & 0 \\
0 & 0 & -1
\end{array}\right),+\left(\begin{array}{ccc}
0 & 0 & 0 \\
0 & 1 & 0 \\
0 & 0 & -1
\end{array}\right)\right) .
\end{aligned}
$$

The matrices are characterized by:

$$
\left[(1 / 2) \lambda_{a},(1 / 2) \lambda_{b}\right]=(i / 2) j_{a b c} \lambda_{c},
$$

where

$$
C_{a b c}:=i j_{a b c}
$$

are the group structure constants of $\mathrm{SU}(3)$ [10]. The structure constants of $\mathrm{SU}(2)$ are

$$
C_{a b c}=i \epsilon_{a b c} .
$$

It can be seen that $\lambda_{2}, \lambda_{5}$, and $\lambda_{7}$ are $\mathrm{O}(3)$ rotation generator matrices, cyclically related by

$$
\left[(1 / 2) \lambda_{2},(1 / 2) \lambda_{5}\right]=(i / 2) j_{257} \lambda_{7}
$$

and this is a representation of three-dimensional Euclidean space with $\mathrm{O}(3)$ group rotation generators. Similarly, $-i \lambda_{1},-i \lambda_{4},-i \lambda_{6}$ are three $\mathrm{O}$ (3) symmetry rotation generator matrices related cyclically by

$$
\left[(1 / 2) \lambda_{1},(1 / 2) \lambda_{4}\right]=(i / 2) j_{146} \lambda_{6}
$$

Finally, the matrices $\lambda_{3}$ and $\lambda_{8}$ are also rotation generator matrices related cyclically by

$$
\left[(1 / 2) \lambda_{3},(1 / 2) \lambda_{8}\right]=(i / 2) j_{38 c} \lambda_{c},
$$

where $c=1,2,4,5,6$, or 7 .

Therefore a rotation in three-dimensional space in $\mathrm{SU}(3)$ representation is given by

$$
q^{\prime}=\exp \left(i \frac{\lambda_{a}}{2} \epsilon_{a}\right) q
$$

an $\mathrm{SU}(3)$ transformation with

$$
q=\left(\begin{array}{c}
q_{R} \\
q_{W} \\
q_{B}
\end{array}\right)
$$


Similarly a rotation in Euclidean, three-dimensional space (the base manifold) can be represented by the $\mathrm{SU}(2)$ transformation

$$
q^{\prime}=\exp \left(i \frac{\sigma^{i}}{2} \epsilon_{i}\right) q
$$

where

$$
q=\left(\begin{array}{l}
q_{1} \\
q_{2}
\end{array}\right)
$$

is the Pauli two-spinor representation of the base manifold using a two-dimensional representation space. In Eq. (36), $q$ is a three-spinor representation of Euclidean three dimensional space.

In order now to represent the strong nuclear field in general relativity, the group indices $a, b, c$ in Eq. (29) are recognized as indices of the orthonormal space of the tetrad, so that Eq. (29) becomes an equation of differential geometry, valid in any spacetime, and independent of the details of the base manifold geometry [4,5]. Similarly, in order to represent the weak nuclear field in general relativity, the indices $a, b, c$ of Eq. (31) become indices of differential geometry. Thus both equations (29) and (31) can be written as

$$
(1 / 2) \lambda_{a} \wedge(1 / 2) \lambda_{b}=(i / 2) j_{a b c} \lambda_{c}
$$

that is,

$$
(1 / 2) q_{a} \wedge(1 / 2) q_{b}=(i / 2) j_{a b c} q_{c}
$$

i.e., become valid in non-Euclidean spacetime as equations of differential geometry. In wedge product notation of differential geometry, Eqs. (30) and (31) define cyclically symmetric relations between wedge products in respectively an $\mathrm{SU}(3)$ and $\mathrm{SU}(2)$ representation of the orthonormal space of the tetrad. In the $\mathrm{SU}(3)$ representation for the strong nuclear field in general relativity the tetrad is defined by the equation

$$
q^{a}=q_{\mu}^{a} q^{\mu}: S U(3)
$$

between a metric three-spinor $q^{a}$ in the orthonormal space labeled $a$ and a metric three-spinor $q^{\mu}$ in the non-Euclidean base manifold, labeled $\mu$. Similarly the tetrad in the SU(2) representation for the weak nuclear field in general relativity is defined by

$$
q^{a}=q_{\mu}^{a} q^{\mu}: S U(2),
$$

where $q^{a}$ is a metric two-spinor in the orthonormal space and where $q^{\mu}$ is a metric two-spinor in the base manifold. In both cases the Evans wave equation is an equation in the tetrad

$$
(\square+k T) q_{\mu}^{a}=0 ;
$$


and in this notation takes the same form for gravitation and for electromagnetism. For both gravitation and electromagnetism, the tetrad is defined by

$$
q^{a}=q_{\mu}^{a} q^{\mu}: O(3)
$$

where $q^{a}$ is a metric four vector in the orthonormal space and where $q^{\mu}$ is a metric four-vector in the base manifold.

It is seen that the strong and weak nuclear fields and the gravitational and electromagnetic field share a common origin in the concept of tetrad, and that the Evans wave equation in all cases is an equation in the tetrad. The nuclear weak field is essentially an $\mathrm{SU}(2)$ representation for the electromagnetic field, and the nuclear strong field an $\mathrm{SU}(3)$ representation of the gravitational field. The Dirac equation is derived [11] from the Evans equation with an SU(2) representation of the gravitational field.

Finally the quark color triplet is a field, so must be a manifestation of the tetrad field. In the standard model only an empirical representation of the color triplet is given, i.e., it is not derived from general relativity and is not identified as a manifestation of a tetrad, the matrix that relates the metrics in the orthonormal space and the base manifold. For each label a of the tetrad, it is a generally covariant object, i.e., a generally covariant four-vector, three-spinor or two-spinor, so the equation governing the gluon field (and all other fields) in general relativity is the Evans equation for each upper index $a$.

Acknowledgments. Craddock, Inc., ADAS and the Ted Annis Foundation are thanked for funding, and the Fellows and Emeriti of the AIAS are thanked for many interesting discussions.

\section{REFERENCES}

1. M. W. Evans, Found. Phys. Lett. 16, 367 (2003).

2. M. W. Evans, "A generally covariant wave equation for grand unified field theory," Found. Phys. Lett. 16, 507 (2003; www.aias.us

3. M. W. Evans, "Equations of unified field theory in terms of the Maurer-Cartan structure relations of differential geometry, Found. Phys. Lett. 17, 25 (2004); www.aias.us

4. S. M. Carroll, Lecture Notes on General Relativity (University of California, Santa Barbara, graduate course on arXiv:gr-qe / 9712019 v1 3 Dec, 1997).

5. R. M. Wald, General Relativity (Chicago University Press, 1984).

6. M. W. Evans, "O(3) Electrodynamics," in M. W. Evans, ed., Modern Nonlinear Optics, a special topical issue in three parts of I. Prigogine and S. A. Rice, series eds, Advances in Chemical Physics (Wiley-Interscience, New York, 2001, second and e-book editions), Vol. 119(2), pp. 79-269. 
7. M. W. Evans and S. Jeffers, "The present status of the quantum theory of light," in Ref. 6, Vol. 119(3), pp. 1-197.

8. M. W. Evans, J.-P. Vigier, et al., The Enigmatic Photon (Kluwer Academic, Dordrecht, 1994 to 2002, hardback and softback editions), Vols 1 to 5 .

9. M. Tatarikis, K. Krushelnik, Z. Najmudin, E. L. Clark, M. Salvati, A. E. Dangor, V. Malka, D. Neely, R. Allott, and C. Danson, "Measurements of the inverse Faraday effect in high intensity laser produced plasmas," CLF Annual Report, Science, High Power Laser Programme, Short Pulse Plasma Physics, Rutherford Appleton Laboratories of the UK EPSRC, 1998/1999, m.tatarikis@ic.ac.uk (Imperial College, London).

10. L. H. Ryder, Quantum Field Theory (Cambridge University Press, 1996).

11. M. W. Evans, "Derivation of the Dirac equation from the Evans field equation," Found. Phys. Lett. 17(2) (2004); www.aias.us 